\title{
BIM INTEGRADO À MINIMIZAÇÃO DA GERAÇÃO DE RESÍDUOS DA CONSTRUÇÃO CIVIL
}

\author{
DE MELO, Alberta Cristina Vasconcelos \\ Instituto Federal do Sertão Pernambucano, Universidade Federal do Mato Grosso do \\ Sul, e-mail: alberta.melo@ifsertao-pe.edu.br \\ SOUZA, Mayara Dias de \\ Universidade Federal do Mato Grosso do Sul, e-mail: mayara.dias@ufms.br \\ CHAGAS, Leila Soares Viegas Barreto \\ Instituto Federal do Sertão Pernambucano, e-mail: leila.viegas@ifsertao-pe.edu.br
}

\begin{abstract}
RESUMO
A indústria da construção civil é bastante relevante no contexto socioeconômico brasileiro e mundial. Contudo, sua atividade caracteriza-se por exercer pressões no meio ambiente, seja pelo consumo de recursos naturais, seja pela geração de somas de resíduos ou por mudanças na paisagem decorrentes do descarte irregular desses. Problemas relacionados a geração de Resíduos da Construção Civil (RCC) se agravam rapidamente e veem sendo debatidos, sendo o discurso centrado em ações de gerenciamento dos RCCs no canteiro de obras ou opções pela reciclagem e/ou reuso, mas poucas experiências foram realizadas utilizando BIM (Building Information Modeling) para minimizar a sua geração. Este artigo faz parte de uma pesquisa de mestrado e restringir-se-á a apresentar uma revisão sistemática da literatura, realizada como etapa inicial da dissertação, analisando a produção científica sobre o uso do BIM na minimização da geração de RCCs, no período de 2009-2017, onde foram selecionadas e analisadas dez publicações relevantes. Utilizou-se métodos de estudos sistemáticos, com natureza descritiva e abordagem qualitativa. Como resultado, identificou-se que o estágio atual do conhecimento acadêmico de BIM na minimização da geração de RCCs está voltado às fases de concepção e projeto e que as pesquisas acadêmicas têm um caráter para propor e validar inovações.
\end{abstract}

Palavras-chave: BIM, Resíduos da Construção Civil, Revisão Sistemática.

\begin{abstract}
The construction industry is very relevant in the Brazilian and global socioeconomic context. However, its activity is characterized by considerable pressure on the environment, both by the consumption of the necessary natural resources and by the sum of the residues generated as a result of its activities, being also responsible for changes in the landscape. The problems related to the generation of Construction Waste (RCC) are quickly increasing and are being debated, but very few experiments were performed using BIM to minimize the generation of RCCs. This article aims to analyze the scientific production on the theme use of BIM, emphasizing the minimization of the generation of construction residues, in the period of 2009-2017. Through a systematic review of literature, 10 (ten) relevant publications were selected and analyzed. Systematic study methods were used, with a descriptive nature and a qualitative approach. As a result, it was identified that the current state of knowledge is delimited on the initials phases: conception and project, and that, the research has a character to propose and validate innovations.
\end{abstract}

Keywords: BIM, Construction Waste, Systematic review.

DE MELO, A. C. V; SOUZA, M. D; CHAGAS, L. S. V. B. BIM integrado à minimização da geração de resíduos da construção civil. In: SIMPÓSIO BRASILEIRO DE QUALIDADE DO PROJETO NO AMBIENTE CONSTRUÍDO, 6., 2019, Uberlândia. Anais... Uberlândia: PPGAU/FAUeD/UFU, 2019. p 647-657. DOI https://doi.org/10.14393/sbqp19060. 


\section{INTRODUÇÃO}

A indústria da construção civil é primordial no atendimento as aspirações da sociedade em geral, ao promover qualidade de vida, reduzir déficits de infraestrutura, gerar empregos, estimular o crescimento e a produção de riquezas para determinado lugar ou região (AGOPYAN; JOHN, 2011). Entretanto, de acordo com o mesmo autor, a indústria da construção civil revela-se como grande geradora de impactos ambientais, quer pelo consumo de recursos naturais, quer pela modificação da paisagem ou ainda pela geração e disposição inadequada de resíduos (KARPINSK et al., 2009).

A participação do RCC de locais como Austrália, Hong Kong, Kuwait, Reino Unido, Estados Unidos e na União Europeia na massa total de resíduos sólidos urbanos (RSU) variava conforme Tabela 1.

Tabela 1 - Participação dos Resíduos de Construção Civil (RCC) anos de 2012 e 2013

\begin{tabular}{|c|c|}
\hline Local & \% em RCC do total de Resíduos Sólidos Urbanos (RSU) produzidos \\
\hline Austrália & $37 \%$ \\
\hline Hong Kong & $38 \%$ \\
\hline Kuwait & $58 \%$ \\
\hline Reino Unido & $60 \%$ \\
\hline Estados Unidos & 10 a $30 \%$ (Estimativa) \\
\hline União Europeia & $31 \%$ \\
\hline
\end{tabular}

Fonte: Elaboração própria (2018), baseado em Brasileiro e Matos (2015)

No Brasil, O RCC representa de 41 a $70 \%$ da massa total de RSU em Salvador/BA e Ribeirão Preto/ SP, respectivamente (PINTO, 1999).

A Agenda 2030, publicada pela Organização Nações Unidas (ONU) em 2015, aponta 17 objetivos e 169 metas para o Desenvolvimento Sustentável (DS). O documento considera diversas realidades nacionais, habilidades e diferentes níveis de desenvolvimento. Neste documento identifica-se o desenvolvimento urbano e a gestão sustentáveis como sendo de fundamental importância para a qualidade de vida das populações e estabelecem, nos objetivos 11 e 12, que até 2030 dentre as demais metas determinadas tem-se a de reduzir o impacto ambiental negativo per capita das cidades, inclusive prestando especial atenção à qualidade do ar, gestão de resíduos municipais e a de reduzir substancial a geração de resíduos por meio da prevenção, redução, reciclagem e reuso, dentre outras ações (ONU, 2015).

O processo de geração e destinação de resíduos, no contexto da construção civil não deveria acontecer de forma linear, tendo o descarte como solução final, sendo que, de acordo com Garé (2011), o ideal é que fossem desenvolvidas ações que atuem na causa, antes da ocorrência dos problemas, considerando nesse processo a opção por estratégias de minimização, reutilização e reciclagem anteriormente a deposição final. $\mathrm{Na}$ Figura 1 tem-se a hierarquia da geração de resíduos, onde as estratégias de não geração e redução são as mais favoráveis e próximas do DS. 


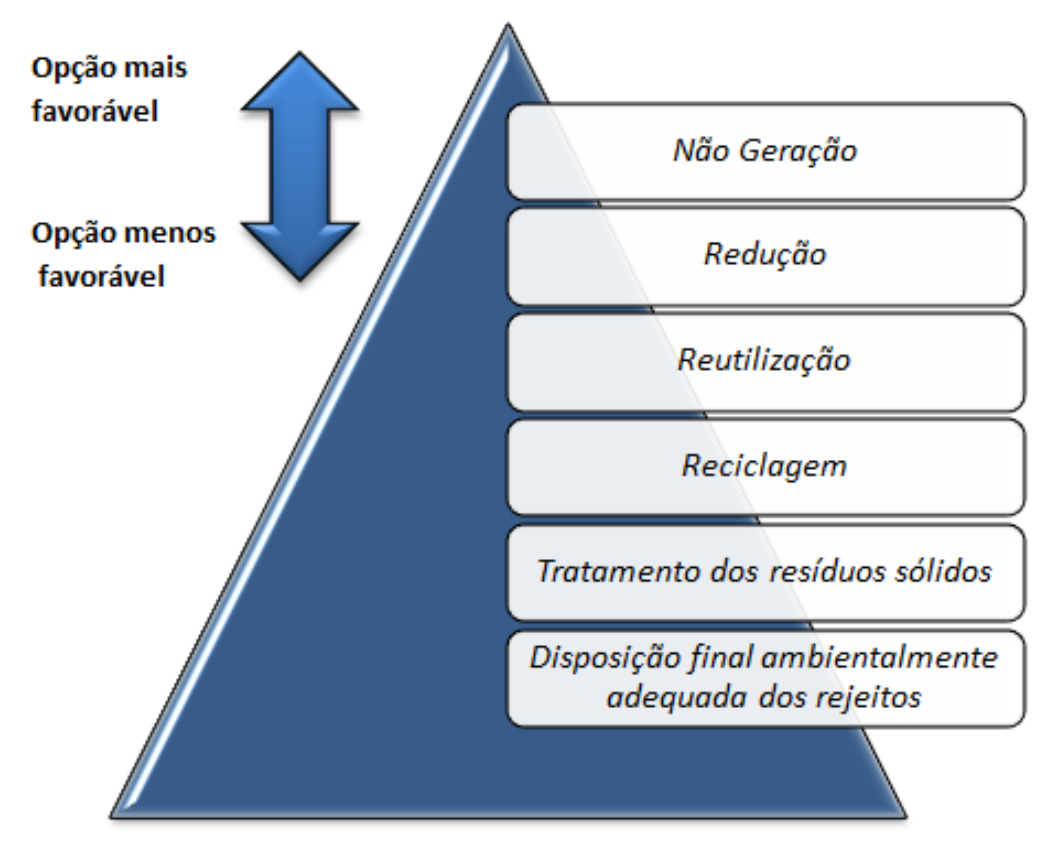

Figura 1 - A hierarquia no gerenciamento de resíduos -

Fonte: <https://www.vgresiduos.com.br/blog/diferenca-destinacao-disposicao-final/>

A falta de motivação pelos profissionais do setor de arquitetura, engenharia e construção para minimizar o desperdício e o baixo nível de reconhecimento da importância da minimização de RCCs levam a problemas na prática do gerenciamento desses (REDMOND et al., 2012).

Won, Cheng e Lee (2016) identificaram o BIM como uma maneira eficaz para diminuir a quantidade gerada dos RCCs, aumentando a qualidade e a precisão do projeto e construção, reduzindo assim os equívocos projetuais, retrabalho e modificações inesperadas.

Diante do contexto apresentado, partiu-se da seguinte questão de pesquisa: como está caracterizada a pesquisa acadêmica sobre a utilização do BIM na Minimização da geração de resíduos da Construção Civile Em decorrência dessa questão o artigo objetiva apresentar uma revisão sistêmica da produção cientifica sobre a utilização do BIM na minimização da geração de resíduos de construção civil, em periódicos com classificação Qualis-Capes: A1 e A2, no período de 2009-2017. Esse artigo está estruturado em quatro partes, além desta Introdução. Inicialmente, é realizada a classificação da pesquisa e o detalhamento do percurso metodológico escolhido, logo após temos análise e discussão dos Resultados, e por fim, as considerações Finais. As referências empregadas são expostas após a quarta seção.

\section{METODOLOGIA}

Utilizou-se o método de Revisão Sistemática com natureza descritiva e abordagem qualitativa, composto por 3 etapas, conforme apresentado no Quadro 1. 
Quadro 1 - Ełapas Revisão Sistemática

\begin{tabular}{|c|l|}
\hline ETAPA 01 & Definição da questão cientifica \\
\hline \multirow{5}{*}{ ETAPA 02 } & $\begin{array}{l}\text { Determinar critério de seleção: adotados os termos relacionadas ao tema } \\
\text { pesquisado, caracterizando as strings de buscas: "BIM" e "construction waste } \\
\text { minimization". }\end{array}$ \\
\cline { 2 - 3 } & $\begin{array}{l}\text { Definição das bases de dados e as estratégias de buscas: Web of Science, } \\
\text { Scopus, ScienceDirect, escolhidas em virtude de serem bases de referência } \\
\text { internacional. }\end{array}$ \\
\hline \multirow{5}{*}{ ETAPA 03 } & $\begin{array}{l}\text { Busca nas bases de dados e seleção dos artigos: Utilizou-se, como ferramenta } \\
\text { de apoio, o software StArt (State of the Art through Systematic Review) pois } \\
\text { contempla as etapas da revisão sistemática: planejamento, execução e } \\
\text { sumarização. }\end{array}$ \\
\cline { 2 - 3 } & $\begin{array}{l}\text { Aplicação dos critérios de seleção dos artigos e justificativas de possíveis } \\
\text { exclusões: (i) abordar ao menos no título, nas palavras-chaves, no resumo os } \\
\text { termos Building Information Modeling e o termo Minimização de RCCs (ii) } \\
\text { disponível em periódicos com acesso livre (iii) intervalo temporal de 2009-2017. }\end{array}$ \\
\cline { 2 - 3 } & $\begin{array}{l}\text { Análise e avaliação dos artigos resultantes da revisão sistemática: verificação } \\
\text { da predominância de periódicos, autores mais citados; autores selecionados; } \\
\text { período de maior incidência de publicações selecionadas. }\end{array}$ \\
\cline { 2 - 3 } & $\begin{array}{l}\text { Apresentação e discussão dos resultados: verificação de predominância de } \\
\text { correlação entre os estudos; identificação dos conjuntos de padrões } \\
\text { relevantes e significantes; temas em aberto para maiores consideração e } \\
\text { abordagens. }\end{array}$ \\
\hline
\end{tabular}

Fonte: Autores (2018)

\section{ANÁLISE DOS DADOS}

A busca retornou 42 artigos para posterior análise, estes atenderam aos critérios de inclusão previamente definidos, abrangendo a temática em debate, colaborando com a discussão da problemática. Ao final do processo de busca foi possível apurar que a produção científica referente ao tema é recente, com a primeiras publicações a partir de 2010, sendo o ano de 2017 o que apresenta maior número de publicações. Com a aplicação dos critérios de inclusão foram selecionados um conjunto de 16 publicações que atenderam simultaneamente aos critérios de inclusão a partir da leitura dos títulos e resumos das publicações. Dentre estes, 06 não correspondiam ao tema em estudo, ou seja, uso do BIM integrado a minimização de RCCs, mas sim a temas semelhantes. Desta maneira, somente 10 trabalhos foram selecionados após a leitura integral dos 16 artigos.

Tabela 2 - Resultado da fase de coleta de dados

\begin{tabular}{|l|c|}
\hline \multicolumn{1}{|c|}{ Fase } & Quantidade \\
\hline Total de artigos & 42 \\
\hline Artigos excluídos & 26 \\
\hline Subtotal 1 & 16 \\
\hline Artigos excluídos após leitura do texto na integra & 6 \\
\hline AMOSTRA FINAL DE ARTIGOS & 10 \\
\hline
\end{tabular}

Fonte: Autores (2018) 
Na Tabela 2 é apresentado o resultado da fase de coleta de dados, que totalizou numa amostra final de 10 artigos.

Após a coleta de dados, que resultou em 10 artigos BIM e a minimização da geração de RCCs, segundo percepção e delimitação da pesquisadora, procedeu-se o mapeamento dos artigos que consistiu em duas etapas: i) análise descritiva e; ii) leitura analítica. Na etapa de análise descritiva, os artigos foram classificados nas seguintes categorias: ano de publicação, número de autores e as revistas científicas em que foram publicadas e suas respectivas áreas e classificação Qualis/Capes. Na Tabela 3 é apresentada a amostra final dos 10 artigos publicados no período de 2009 a 2017.

\section{Tabela 3 - Amostra final dos artigos por ordem cronológica de publicação, os respectivos autores, títulos, palavras-chave, país de origem dos autores e as revistas dos artigos selecionados}

\begin{tabular}{|c|c|c|c|}
\hline N. & Autor & Título & País \\
\hline 01 & Cheng e Ma (2013) & $\begin{array}{l}\text { A BIM-based system for demolition and } \\
\text { renovation waste estimation and planning }\end{array}$ & China \\
\hline 02 & Gurevich e Sacks (2014) & $\begin{array}{l}\text { Examination of the effects of a KanBIM } \\
\text { production control system on } \\
\text { subcontractors' task selections in interior } \\
\text { works }\end{array}$ & Israel \\
\hline 03 & Li e Yang (2014) & $\begin{array}{l}\text { Critical factors for waste management in } \\
\text { office building retrofit projects in Australia }\end{array}$ & Austrália \\
\hline 04 & $\begin{array}{l}\text { Liu, Osmani, Demian e } \\
\text { Baldwin (2015) }\end{array}$ & $\begin{array}{l}\text { A BIM-aided construction waste } \\
\text { minimisation framework }\end{array}$ & Reino Unido \\
\hline 05 & $\begin{array}{l}\text { Akinade, Oyedele, Bilal, } \\
\text { Ajayi, Owolabi, Alaka e } \\
\text { Bello (2015) }\end{array}$ & $\begin{array}{l}\text { Waste minimisation through deconstruction: } \\
\text { A BIM based Deconstructability Assessment } \\
\text { Score (BIM-DAS) }\end{array}$ & $\begin{array}{l}\text { Reino Unido; } \\
\text { Nigéria }\end{array}$ \\
\hline 06 & $\begin{array}{l}\text { Ajayi, Oyedele, Bilal, } \\
\text { Akinade, Alaka, Owolabi } \\
\text { e Kadiri (2015) }\end{array}$ & $\begin{array}{l}\text { Waste effectiveness of the construction } \\
\text { industry: Understanding the impediments } \\
\text { and requisites for improvements }\end{array}$ & $\begin{array}{l}\text { Reino } \\
\text { Unido; } \\
\text { Nigéria }\end{array}$ \\
\hline 07 & $\begin{array}{l}\text { Won, Cheng e Lee } \\
(2016)\end{array}$ & $\begin{array}{l}\text { Quantification of construction waste } \\
\text { prevented by BIM-based design validation: } \\
\text { Case studies in South Korea }\end{array}$ & $\begin{array}{l}\text { China; } \\
\text { Coréia do Sul }\end{array}$ \\
\hline 08 & Won e Cheng (2017) & $\begin{array}{l}\text { Identifying potential opportunities of } \\
\text { building information modeling for } \\
\text { construction and demolition waste } \\
\text { management and minimization }\end{array}$ & $\begin{array}{l}\text { China; } \\
\text { Coréia do Sul }\end{array}$ \\
\hline 09 & $\begin{array}{l}\text { Alwan, Jones e Holgate } \\
\text { (2017) }\end{array}$ & $\begin{array}{l}\text { Strategic sustainable development in the UK } \\
\text { construction industry, through the framework } \\
\text { for strategic sustainable development, using } \\
\text { Building Information Modelling }\end{array}$ & Reino Unido \\
\hline 10 & $\begin{array}{l}\text { Lu, Webster, Chen, } \\
\text { Zhang e Chen (2017) }\end{array}$ & $\begin{array}{l}\text { Computational Building Information } \\
\text { Modelling for construction waste } \\
\text { management: Moving from rhetoric to } \\
\text { reality }\end{array}$ & China \\
\hline
\end{tabular}

Fonte: Autores (2018)

Os 10 artigos estão publicados em Periódicos. A Figura 2 apresenta quais são eles e a quantidade de artigos publicados em cada um deles. Observa-se que a maioria das publicações aconteceu em dois Periódicos: Resources, Conservation and Recycling e Automation in Construction cada um publicou três artigos sobre o tema. 


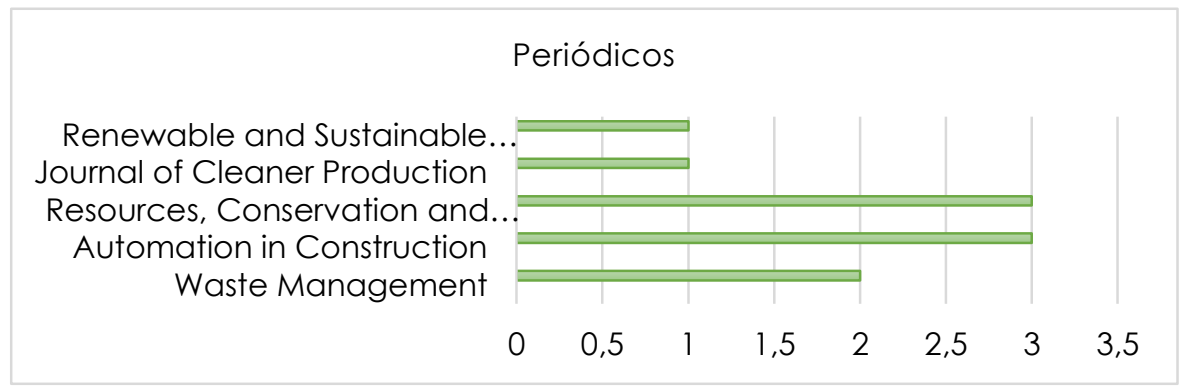

Figura 2 - Periódicos científicos de todos os artigos selecionados Fonte: Autores (2018)

De acordo com análise observou-se que não há concentração de publicações em um ano específico. Contudo, constatou-se que a maior ocorrência de publicações se deu em 2017, tal como demonstra a Figura 3. Pode-se constatar que o assunto é recente e com grandes possibilidades de campos a serem explorados.

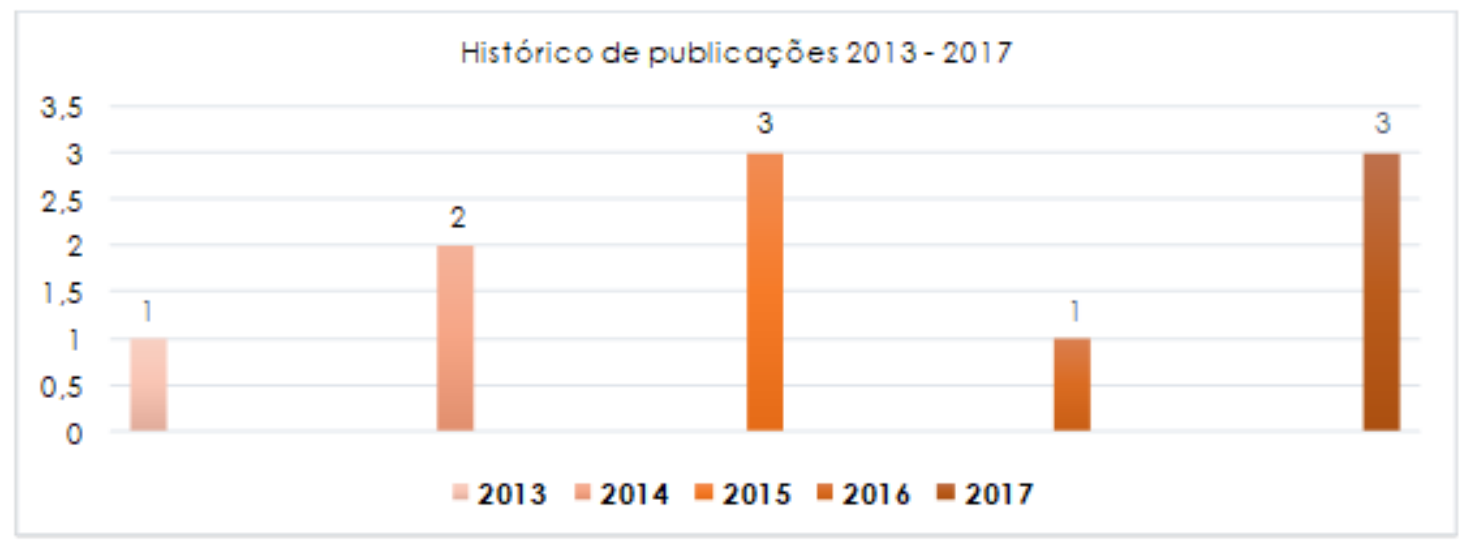

Figura 3 - Histórico de publicações no período entre 2013-2017 -

Fonte: Autores (2018)

A maior ocorrência de artigos se deu em periódicos da área de Engenharias I, o que demonstra que o tema BIM está inserido e vinculado à temática dos resíduos. Evidencia-se que todos as publicações selecionadas por este estudo obtiveram avaliação máxima - A1, na classificação Qualis-Capes, que indica qualidade superior nas publicações.

Dos 37 autores levantados, 16 deles publicaram em mais de um artigo sobre o tema no período pesquisado.

Com relação a nacionalidade dos pesquisadores nota-se que a maior concentração é do Reino Unido, sendo quatro o número de autores das publicações analisadas. Considerou-se apenas a nacionalidade do autor principal como referência, sem considerar a dos co-autores, tendo em vista que em alguns artigos eram de outras nacionalidades.

As buscas não apontaram trabalhos de autores brasileiros sobre o uso de BIM na minimização de geração dos RCCs e/ou objetivando a quantificação deles, pode-se refletir que essas proposições de uso e aplicação do BIM ainda sejam pouco conhecidas no Brasil tendo em vista que a utilização do BIM ainda é um processo recente. 
O tema do uso do BIM na minimização da geração de RCC está em discussão por pesquisadores de países da Ásia (China, Coréia do Sul e Israel), da Oceania (Austrália) e da Europa (Reino Unido). A Figura 4 mostra a incidência das nacionalidades dos autores.

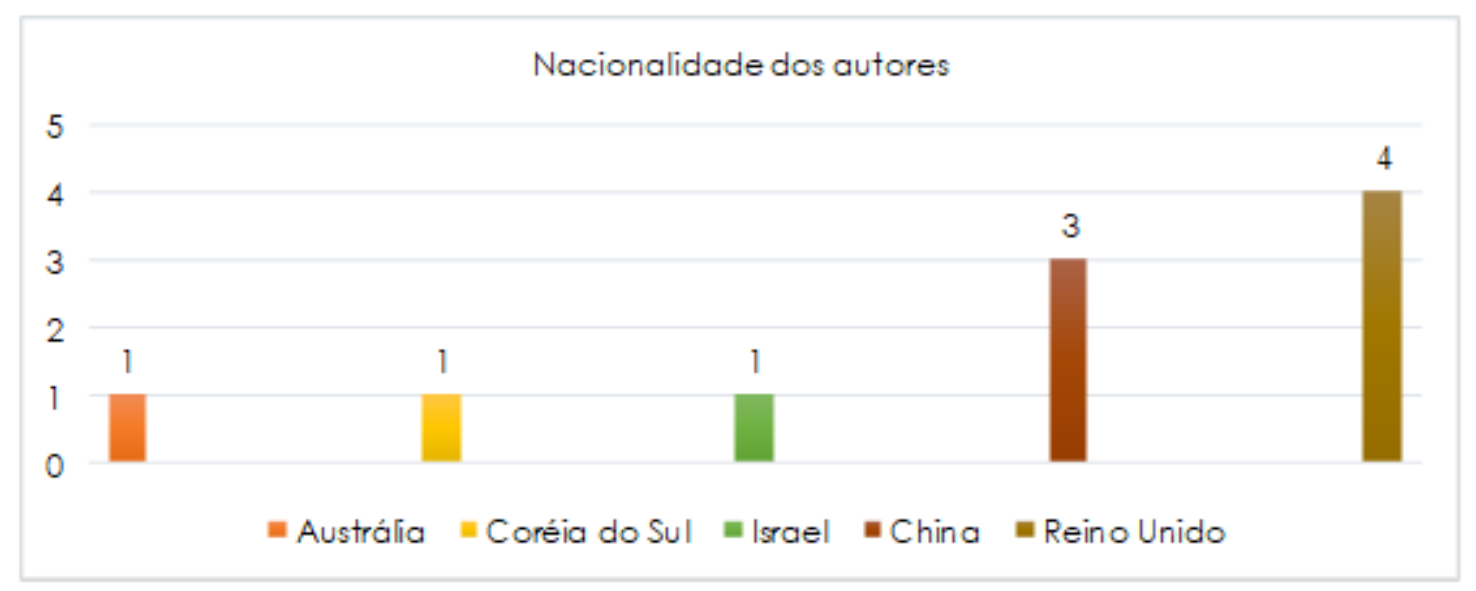

Figura 4 - Nacionalidade dos autores -

Fonte: Autores (2018)

\subsection{Leitura Analítica}

Com relação à leitura analítica, buscou-se identificar, dentre as pesquisas avaliadas, quais os tipos de métodos foram utilizados, sendo estes (Figura 5): 40\% Pesquisa Bibliográfica, 50\% Pesquisa Experimental e 10\% Estudo de Caso. Esta análise demonstra que as pesquisas voltam seu olhar para a proposição e validação de inovações (experimentos e construtivos) sendo pouco abordada a prática (estudos de casos).

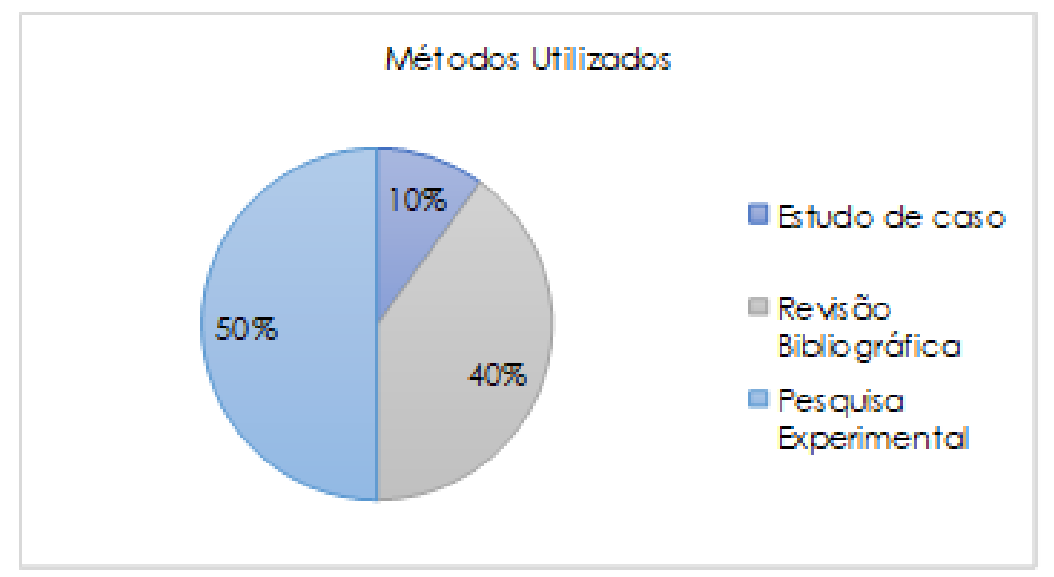

Figura 5 - Métodos utilizados -

Fonte: Autores (2018)

Através desta análise pode-se refletir que as ferramentas e métodos existentes para tratar da minimização da geração de resíduos são insuficientes e que o BIM oferece a oportunidade de preencher essa lacuna, como observado por Cheng e Ma (2013).

O BIM é aplicado nas fases do ciclo de vida de uma edificação: concepção, projeto, construção, operação, retrofit e/ou demolição, observa-se que em relação a aplicação dos sistemas baseados em BIM das pesquisas experimentais e de estudo de caso, que a maioria dos sistemas propostos 
apresentou maior ênfase da integração na fase de concepção e projeto (Figura 6). Tal fato pode ser explicado dado que o BIM é capaz de fornecer um ambiente computacional, virtual e menos dispendioso para refletir sobre várias opções de projeto e esquemas de construção, ambos com o objetivo de minimizar o desperdício (LU et al., 2017).

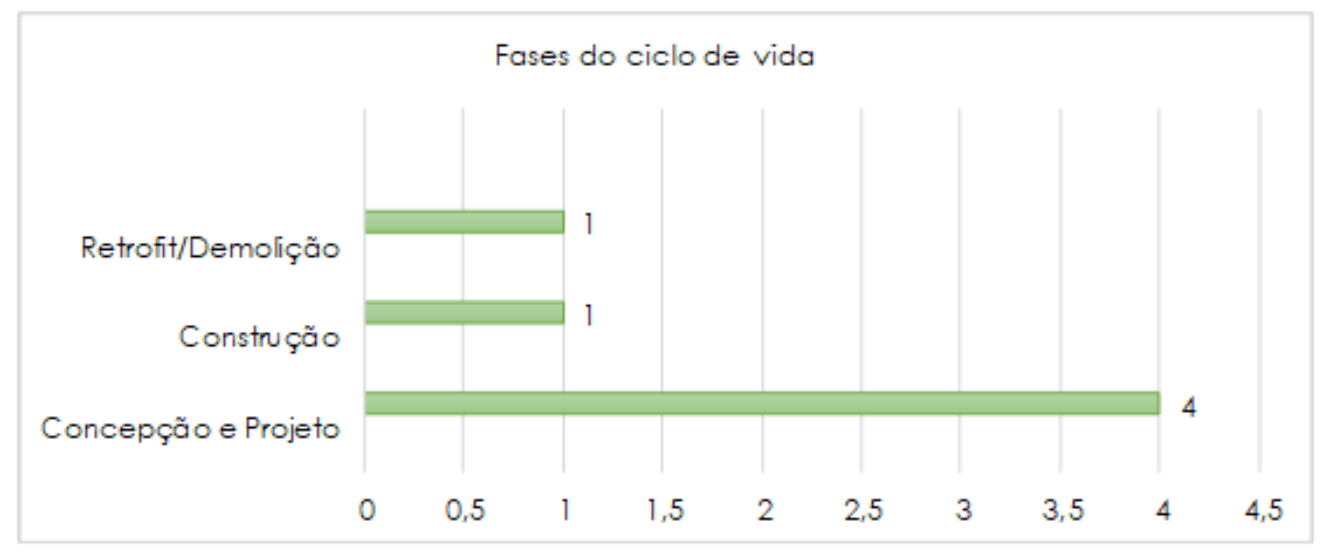

Figura 6 - Fases do ciclo de vida -

Fonte: Autores (2018)

Embora a maioria das pesquisas analisadas apontem para a utilização do BIM na minimização da geração dos RCCs em fases de concepção e projeto, Won e Cheng (2017), afirmam que a utilização do BIM para gestão de resíduos pode acontecer nas fases de planejamento, projeto, construção e demolição de empreendimentos. Sendo o KanBIM, neste contexto, utilizado para promover a discussão entre líderes de equipes sobre tarefas a serem executadas no canteiro de obras (GUREVICH; SACKS, 2014).

Neste contexto Li e Yang (2014) citam ainda em seu estudo bibliográfico que a digitalização a laser 3D e o Scan-to-BIM, foram desenvolvidas para o mercado de construção australiano para realizar levantamento otimizado de construções já existentes, favorecendo a produção de desenhos as-built precisos e a construção de modelos 3D BIM de modo a facilitar a compreensão das questões de gestão de RCCs em projetos de retrofit e fornecer uma referência para os profissionais do setor (AEC) desenvolverem medidas relevantes com relação a esta problemática.

Dentre as pesquisas avaliadas buscou-se identificar também quais as propriedades do BIM foram aplicadas no desenvolvimento dos sistemas, que se relacionam com os conceitos, características e aplicações BIM: a utilização da parametrização de dados (Cheng e Ma, 2013; Akinade et al., 2015), de realidade virtual (GUREVICH; SACKS, 2014), de detecção de interferências (WON; CHENG; LEE, 2016) este utiliza método de validação de projeto que considera o tipo de erro e a probabilidade de identificar erros sem o BIM.

Destaca-se, contudo a pesquisa de Akinade et al. (2015) que utiliza de modelagem matemática no desenvolvimento do sistema de Avaliação de Desconstrutabilidade - BIM-DAS.

E em outro estudo a utilização de algoritmos BIM computacionais para manipular e fornecer informações de tomada de decisão para minimização da geração de RCCs, como opções de projeto de baixo desperdício e/ou esquemas de construção (LU, et al., 2017). 
No Tabela 4 é apresentada a amostra dos 06 artigos que abordam métodos de pesquisa experimental e estudo de caso e as propriedades do BIM aplicadas no desenvolvimento dos sistemas.

\section{Tabela 4 - Apresenta os artigos por ordem cronológica de publicação, os respectivos autores, sistemas desenvolvidos e propriedade BIM aplicada dos seis artigos que tratam de pesquisa experimental e estudo de caso}

\begin{tabular}{|c|c|c|}
\hline Autor & Sistema desenvolvido & Propriedade BIM \\
\hline Cheng e Ma (2013) & $\begin{array}{c}\text { Sistema baseado em BIM para } \\
\text { estimativa e planejamento de RCC. }\end{array}$ & $\begin{array}{l}\text { Parametrização de } \\
\text { dados }\end{array}$ \\
\hline $\begin{array}{l}\text { Gurevich e Sacks } \\
(2014)\end{array}$ & $\begin{array}{l}\text { KanBIM - Sistema de controle de } \\
\text { produção }\end{array}$ & Realidade Virtual \\
\hline Liv et al. (2015) & BaW Framework & $\begin{array}{l}\text { Plataforma integrada } \\
\text { ao BIM }\end{array}$ \\
\hline Akinade et al. (2015) & $\begin{array}{l}\text { BIM-DAS - Sistema de Avaliação de } \\
\text { Desconstrutabilidade baseado em BIM; }\end{array}$ & $\begin{array}{l}\text { Parametrização de } \\
\text { dados }\end{array}$ \\
\hline $\begin{array}{l}\text { Won, Cheng e Lee } \\
\text { (2016) }\end{array}$ & $\begin{array}{c}\text { Método por validação de projeto que } \\
\text { considera o tipo de erro e a } \\
\text { probabilidade de identificar erros sem o } \\
\text { BIM }\end{array}$ & $\begin{array}{l}\text { Detecção de } \\
\text { interferências }\end{array}$ \\
\hline Lu et al. (2017) & $\begin{array}{l}\text { Desenvolvimento de algoritmos BIM } \\
\text { computacionais }\end{array}$ & $\begin{array}{l}\text { Utilização de algoritmos } \\
\text { BIM computacionais }\end{array}$ \\
\hline
\end{tabular}

Fonte: Autores (2018)

Observa-se que em relação aos benefícios, os estudos de Cheng e Ma (2013) e Lu et al. (2017) demonstraram que os sistemas desenvolvidos foram capazes de gerar informações detalhadas sobre volumes de resíduos gerados, e estimar a quantidade potencial dos mesmos, além de que Lu et al. (2017), observa que a pesquisa em taxas de geração de resíduos ajudará a desenvolver as principais informações, estas organizadas em bancos de dados autônomos mas conectadas a BIM ou encapsuladas nos componentes BIM tornando suas essas novas propriedades.

Em específico na pesquisa de Won, Cheng e Lee (2016) tem-se que o método utilizado avaliou que de 4,3 a 15,2\% dos RCCs poderiam ter sido gerados caso não se utilizasse o BIM, o estudo, portanto foi apontando como eficaz para evitar a geração de resíduos de construção no local. No estudo de Akinade et al. (2015), a abordagem ajudou a caracterizar os materiais de construção e suas propriedades de modo que, dado um projeto BIM, o modelo matemático pode avaliar seu desempenho no projeto de desconstrução, atribuindo uma pontuação BIM-DAS ao projeto.

Os benefícios apontados por Liu et al. (2015) observam que BaW Framework fornece um processo de tomada de decisão de minimização da geração dos RCCs integrado durante o projeto, e que o sistema poderia ser adotado e customizado por outras disciplinas de projeto de construção, como projetos estruturais, por exemplo.

Com relação as desvantagens e ou limitações tem-se que a pesquisa de Ajay et al. (2015) apresentou limitações com relação a finalidade do estudo, restringindo-se a dados qualitativos, e ao contexto do Reino Unido, na pesquisa de Li e Yang (2014) a população alvo da amostra, também é pequena devido ao foco específico em projetos de retrofit de edifícios de 
escritórios da Austrália. Já no sistema KanBIM as limitações observadas são referentes ao fato que a fixação da tela para o acesso das informações está em um único lugar no canteiro de obras e da impossibilidade de se preencher os critérios de qualidade no próprio sistema (GUREVICH; SACKS, 2014), o sistema desenvolvido por Cheng e Ma (2013) não articulou as principais informações necessárias para desenvolver um algoritmo convincente para realizar a estimativa com base nas informações especificadas e, por fim no estudo de Won, Cheng e Lee (2016), que baseou-se em erros evitáveis, apresenta um pequeno número de casos, limitando-se a 2 casos.

\section{CONSIDERAÇÕES FINAIS}

Por conta do delineamento de pesquisa proposto, acredita-se que o objetivo deste levantamento sistemático foi alcançado, pois os resultados permitiram conhecer e mapear as características dos artigos publicados sobre a pesquisa acadêmica a respeito da utilização do BIM na minimização da geração de RCCs, tendo em vista a relevância e importância do tema.

Em função do método de pesquisa aplicado, foi possível verificar que do total de 42 artigos levantados, inicialmente, somente 10 referiram-se aos termos de pesquisa correlacionados com o uso do BIM e a minimização de RCCs, ou seja, um índice de apenas $23,80 \%$ de êxito na busca realizada, de modo que é possível constatar a pouca quantidade de estudos acerca do tema.

Em relação aos métodos utilizados destacou-se o emprego da pesquisa experimental. Esta análise demonstra que as pesquisas voltam seu olhar para a proposição e validação de inovações.

Em relação as propriedades do BIM aplicadas no desenvolvimento dos sistemas, se observou que os estudos analisados indicam utilização de diversos conceitos: de parametrização, de realidade virtual, de detecção de interferências, plataformas integradas e algoritmos computacionais.

Conclui-se, dessa forma, que o estágio atual do conhecimento acadêmico de BIM na Minimização da geração de RCCs está voltado às fases de concepção e projeto e que as pesquisas acadêmicas têm caráter para propor e validar inovações.

\section{REFERÊNCIAS}

AGOPYAN, V.; JOHN, V. M. O Desafio da Sustentabilidade na Construção Civil. São Paulo: Blucher, 2011

AJAYI, S. O.; BILAL, A.; ALAKA, O., K. Waste effectiveness of the construction industry: understanding the impediments and requisites for improvements. Resources, Conservation and Recycling, v. 102, p.101-112, set. 2015.

AKINADE, O. O.; OYEDELE, L. O.; BILAL, M.; AJAYI, S. O.; OWOLABI, H. A.; ALAKA, H. A.; BELLO, S. A. Waste minimisation through deconstruction: a BIM based Deconstructability Assessment Score (BIM-DAS). Resources, Conservation and Recycling, v. 105, p.167-176, dez. 2015.

ALWAN, Z.; JONES, P.; HOLGATE, P. Strategic sustainable development in the UK construction industry, through the framework for strategic sustainable 
development, using Building Information Modelling. Journal of Cleaner Production, [s.l.], v. 140, p.349-358, jan. 2017. Elsevier BV.

BRASILEIRO, L. L.; MATOS, J. M. E. Revisão bibliográfica: reutilização de resíduos da construção e demolição na indústria da construção civil. Cerâmica 61, 178189, 2015.

GARÉ, J. C. Contribuições da construção civil brasileira para o desenvolvimento sustentável. 2011. Dissertação (Mestrado) - Curso de Administração, Universidade Municipal de São Caetano do Sul, São Caetano do Sul, 2011.

CHENG, J.; MA, L. A BIM-based system for demolition and renovation waste estimation and planning. Waste Management, [s.I.], v. 33, n. 6, p.1539-1551, jun. 2013.

GUREVICH, U.; SACKS, R. Examination of the effects of a KanBIM production control system on subcontractors' task selections in interior works. Automation in Construction, v. 37, p.81-87, jan. 2014.

KARPINSK, L. A.; PANDOLFO, A.; REINEHR, R.; KUREK J.; PANDOLFO, L.; GUIMARÃES, J. Gestão diferenciada de resíduos da construção civil: uma abordagem ambiental [recurso eletrônico] Porto Alegre: Edipucrs, 2009. Disponível em: <http://www.sinduscondf.org.br/portal/arquivos/GestaodeResiduosPUCRS.pdf>. Acesso em: 20 out. 2018.

LI, M.; YANG, J. Critical factors for waste management in office building retrofit projects in Australia. Resources, Conservation and Recycling, v.93, p.85-98, dez. 2014.

LIU, Z.; OSMANI, M.; DEMIAN, P.; BALDWIN, A. A BIM-aided construction waste minimisation framework. Automation in Construction, v.59, p.1-23, nov. 2015.

LU, W.; WEBSTER, C.; CHEN, K.; ZHANG, X. Computational Building Information Modelling for construction waste management: moving from rhetoric to reality. Renewable and Sustainable Energy Reviews, v. 68, p.587-595, fev. 2017.

ORGANIZAÇÃO DAS NAÇÕES UNIDAS. Transformando nosso mundo: A Agenda 2030 para o Desenvolvimento Sustentável. 2015. Disponível em: <https://nacoesunidas.org/pos2015/>. Acesso em: 23 out. 2018.

PINTO, T. P. Metodologia para a gestão diferenciada de resíduos sólidos da construção urbana. São Paulo, 1999. Tese (Doutorado) - Escola Politécnica da Universidade de São Paulo, São Paulo, 1999.

REDMOND, A.; HORE, A.; ALSHAWI, M.; WEST, R. Exploring how information exchanges can be enhanced through Cloud BIM. Automation in Construction, v.24, p.175-183. jul. 2012. Elsevier BV.

WON, J; CHENG, J. C. Identifying potential opportunities of building information modeling for construction and demolition waste management and minimization. Automation in Construction, [s.I.], v. 79, p.3-18, jul. 2017.

WON, J; CHENG, J.; LEE, G. Quantification of construction waste prevented by BIM-based design validation: Case studies in South Korea. Waste Management, v.49, p.170-180, mar. 2016. 